\title{
OPEN Evaluating very high energy electron RBE from nanodosimetric pBR322 plasmid DNA damage
}

\author{
K. L. Small ${ }^{1,2}$, N. T. Henthorn ${ }^{3,4}$, D. Angal-Kalinin ${ }^{1,2,7}$, A. L. Chadwick ${ }^{3,4}$, E. Santina ${ }^{3,4}$, \\ A. Aitkenhead ${ }^{3,5}$, K. J. Kirkby ${ }^{3,4}$, R. J. Smith ${ }^{2,7}$, M. Surman ${ }^{2,7}$, J. Jones ${ }^{2,7}$, W. Farabolini, ${ }^{6,8}$, \\ R. Corsini ${ }^{6}$, D. Gamba ${ }^{6}$, A. Gilardi, ${ }^{6,9}$, M. J. Merchant ${ }^{3,4}$ \& R. M. Jones ${ }^{1,2}$
}

This paper presents the first plasmid DNA irradiations carried out with Very High Energy Electrons (VHEE) over 100-200 MeV at the CLEAR user facility at CERN to determine the Relative Biological Effectiveness (RBE) of VHEE. DNA damage yields were measured in dry and aqueous environments to determine that $~ 99 \%$ of total DNA breaks were caused by indirect effects, consistent with other published measurements for protons and photons. Double-Strand Break (DSB) yield was used as the biological endpoint for RBE calculation, with values found to be consistent with established radiotherapy modalities. Similarities in physical damage between VHEE and conventional modalities gives confidence that biological effects of VHEE will also be similar-key for clinical implementation. Damage yields were used as a baseline for track structure simulations of VHEE plasmid irradiation using GEANT4-DNA. Current models for DSB yield have shown reasonable agreement with experimental values. The growing interest in FLASH radiotherapy motivated a study into DSB yield variation with dose rate following VHEE irradiation. No significant variations were observed between conventional and FLASH dose rate irradiations, indicating that no FLASH effect is seen under these conditions.

In the UK, $27 \%$ of cancer patients receive radiotherapy as part of their treatment ${ }^{1}$, primarily with $12 \mathrm{MV}$ X-rays though proton therapy treatments are increasing ${ }^{2}$. In the past two decades, developments in high-gradient linear accelerator technology $y^{3,4}$ has motivated research into the use of Very High Energy Electrons (VHEE), typcially defined as electrons in the energy range $100-250 \mathrm{MeV}$, as a radiotherapy modality ${ }^{5}$. By adapting existing highgradient accelerator technology from linear colliders for high energy particle physics, medical accelerators with accelerating gradients of $\sim 100 \mathrm{MV} / \mathrm{m}$ could be capable of producing $250 \mathrm{MeV}$ electrons with an accelerator length of 3-4 m.

VHEE radiotherapy has been shown to exhibit potential advantages such as sufficient penetrative range to treat deep-seated tumours, reduced lateral penumbra, relative insensitivity to tissue inhomogeneities ${ }^{6}$ and rapid treatment delivery ${ }^{7}$. This makes VHEE an exciting potential radiotherapy modality and particularly applicable for tumours in highly heterogeneous regions such as the lung. The ability to deliver treatment rapidly makes VHEE a compatible modality for ultra-high dose rate radiotherapy (>40 Gy/s), referred to as FLASH radiotherapy ${ }^{8}$. At such high dose rates, side effects in normal, healthy tissue have been shown in several in vivo models to be drastically reduced while tumour control rates are maintained ${ }^{9-11}$. Although Bourhis et al..$^{12}$ have presented the first patient treatment by FLASH with favourable outcomes, further understanding of the mechanisms and longterm effects are required before widespread clinical implementation. Combining FLASH therapy with VHEE could provide a potential method to treat tumours in heterogeneous regions while exploiting the benefits of the FLASH effect ${ }^{13}$.

The primary mechanism behind radiotherapy is considered to be DNA damage. Ionising radiation can cause direct or indirect damage to DNA: direct damage is caused by energy deposition from the radiation directly to the DNA structure while indirect damage is caused by free radical attack following the dissociation of water

\footnotetext{
${ }^{1}$ The University of Manchester, Manchester, UK. ${ }^{2}$ The Cockcroft Institute, Daresbury, UK. ${ }^{3}$ Division of Cancer Sciences, School of Medical Sciences, Faculty of Biology, Medicine and Health, The University of Manchester, Manchester, UK. ${ }^{4}$ The Christie NHS Foundation Trust, Manchester Academic Health Science Centre, Manchester, UK. ${ }^{5}$ Christie Medical Physics and Engineering, The Christie NHS Foundation Trust, Manchester, UK. ${ }^{6} \mathrm{CERN}$, Geneva, Switzerland. ${ }^{7}$ ASTeC, STFC Daresbury Laboratory, Daresbury, Warrington, UK. ${ }^{8}$ CEA Saclay, IRFU-DACM, Saclay, France. ${ }^{9}$ Federico II, DIETI, University of Napoli, Napoli, Italy. ${ }^{\circledR}$ email: kristina.small@manchester.ac.uk
} 


\begin{tabular}{|l|l|l|l|}
\hline Sample type & SC & OC & L \\
\hline Control (dry) & $0.867 \pm 0.004$ & $0.100 \pm 0.003$ & $0.033 \pm 0.001$ \\
\hline Sham (dry) & $0.270 \pm 0.013$ & $0.690 \pm 0.011$ & $0.038 \pm 0.002$ \\
\hline Control (wet) & $0.877 \pm 0.008$ & $0.096 \pm 0.006$ & $0.027 \pm 0.002$ \\
\hline Sham (wet) & $0.882 \pm 0.008$ & $0.091 \pm 0.007$ & $0.027 \pm 0.001$ \\
\hline
\end{tabular}

Table 1. Comparison of the proportions of supercoiled (SC), open-circular (OC) and linear (L) plasmid in control and sham (unirradiated samples transported to and from CLEAR) plasmid samples. Standard error based on three gel electrophoresis repeats and four control samples for each gel.

molecules by the radiation, in particular $\mathrm{OH}^{-}$due to their high reaction rate with DNA components ${ }^{14}$. Indirect damage is the main contributor to the total damage following exposure to low Linear Energy Transfer (LET) radiation ${ }^{15}$. The cell is equipped with a complex machinery to attempt to resolve this damage, with SingleStrand Breaks (SSBs) and Double-Strand Breaks (DSBs) ${ }^{16}$ being most difficult to repair. If these breaks are not repaired or are misrepaired, the cell may be unable to function or replicate correctly, potentially leading to cell death or senescence ${ }^{17}$. If physical damage resulting from VHEE irradiation is comparable to damage caused by traditional radiotherapy modalities, this will give confidence that the chemical and biological effects of VHEE are also comparable.

For successful clinical implementation of VHEE, a thorough radiobiological understanding is required along with comparison to well-established radiotherapy modalities through Relative Biological Effectiveness (RBE) to determine if dose prescription for VHEE requires biological augmentation. RBE is defined as the ratio of biological effectiveness of one type of ionizing radiation relative to another, conventionally ${ }^{60} \mathrm{Co} \mathrm{X}$-rays, given the same amount of absorbed energy (dose) ${ }^{18}$. This is measured using several endpoints, including DNA damage and, most commonly, cell survival ${ }^{19}$.

This study presents the first pBR322 plasmid irradiations with VHEE beams, over a clinically relevant energy range. Plasmids are ring-like structures of DNA found in bacteria ${ }^{20}$ and were employed to investigate the potential of VHEE to induce DNA damage due to their lack of repair mechanism and ability to study in aqueous and dry environments, allowing the decoupling of direct and indirect damage. Irradiations were carried out at the CERN Linear Electron Accelerator for Research (CLEAR) facility ${ }^{21,22}$. SSB and DSB yields were measured to determine how DNA damage varied with energy and environment. The DSB yields following both dry and aqueous irradiation were used as the biological endpoint for the calculation of VHEE RBE, which was compared to RBE of other radiotherapy modalities. A parameter variation study was then performed for a GEANT4-DNA plasmid irradiation model to determine the parameters which would result in DSB yields which best approximated the experimental data.

The capability of CLEAR to deliver radiation at ultra-high dose rates through ps pulses also allowed the investigation of damage caused to aqueous plasmid samples following irradiation at ultra-high and conventional dose rates. While there is a great deal of research available on ultra-high dose rate irradiation dating back to the $1960 \mathrm{~s}^{23}$, it typically involves cellular irradiation ${ }^{24-29}$. This study focuses on plasmid irradiation at conventional and ultra-high dose rates to determine the presence of a FLASH effect at the nanoscale. Such an effect would be expected to be a decrease in DNA damage yields however, as plasmid irradiation experiments lack many of the key features that lead to the FLASH mechanism, it was not expected that such a decrease would be observed.

\section{Results}

Plasmid stability during transportation. For dry plasmid samples, the diluted plasmid solution was transported to CERN and dry samples prepared onsite. For wet samples, a shipment of pBR322 plasmid, held in a solution of $10 \mathrm{mM}$ Tris- $\mathrm{HCl}$ and $1 \mathrm{mM} \mathrm{EDTA}^{30,31}$, was sent from New England BioLabs to CERN. This was then diluted and wet samples prepared onsite. For both wet and dry samples, control samples were prepared and stored at the Oglesby Cancer Research Centre.

Sham samples were prepared at CERN, mounted on to the sample holder but not directly exposed to the beam. Supercoiled (SC), open-circular (OC) and linear (L) plasmid proportions for control and sham samples were compared to determine the effect of transportation, preparation and indirect radiation exposure. The results are presented in Table 1 .

The plasmid proportion data indicates that transportation in dilute solution did have a significant effect on the dry plasmid samples, with high proportions of open-circular plasmid observed compared to the control samples-indicating a relaxing of the plasmid structure, resulting in SSBs that are not caused by irradiation. Transportation had little effect on the linear plasmid proportion. The effects of transportation were not as severe on the aqueous samples due to transportation in undiluted buffer, which prevented the relaxation of the plasmid structure. Comparison of the dry and wet control samples indicate that the plasmid drying process does not result in significant damage to the plasmid structure.

Dry sample irradiations. The damage yields, calculated using the McMahon DNA damage fit ${ }^{32}$, over 100$200 \mathrm{MeV}$ are shown in Fig. 1a, b and in Table 2 below, with standard errors calculated based on three Agarose Gel Electrophoresis (AGE) repeats. 

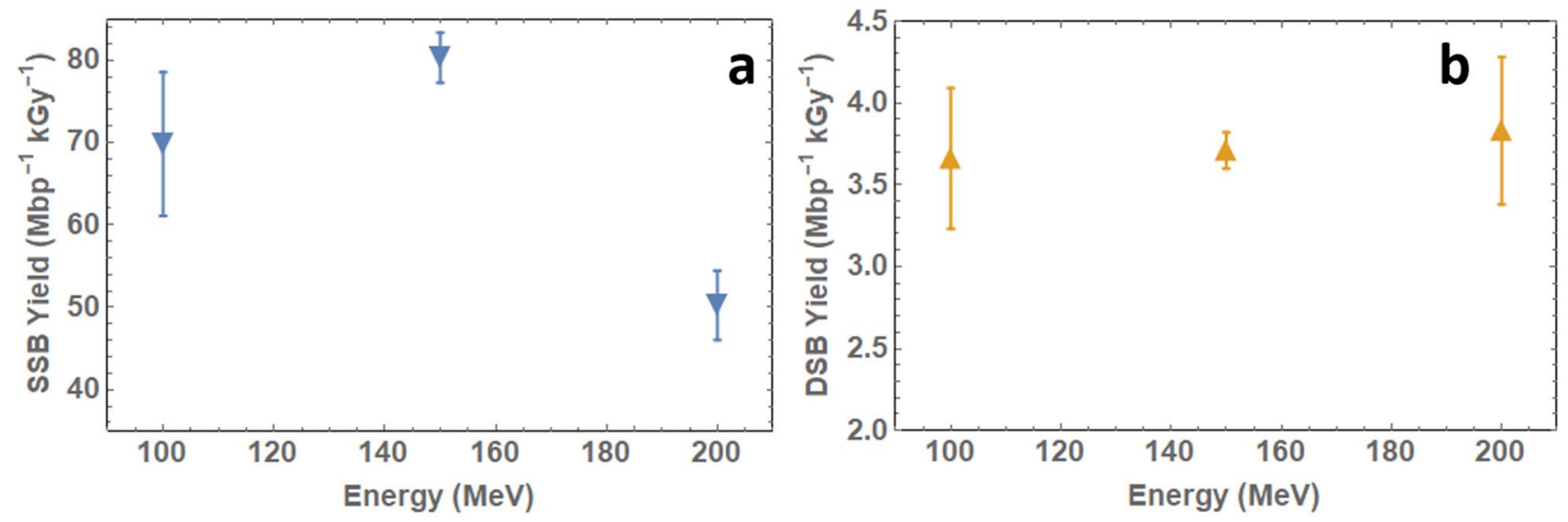

Figure 1. (a) Single-strand break yields and (b) double-strand break yields for 100-200 MeV electron beam irradiation of dry pBR322 plasmid DNA based on McMahon data fitting ${ }^{32}$.

\begin{tabular}{|l|l|l|l|}
\hline Energy $(\mathbf{M e V})$ & LET $(\mathbf{k e V} / \mu \mathrm{m})$ & SSB $\left(\mathbf{M b p}^{-\mathbf{1}} \mathbf{k G \mathbf { y } ^ { - 1 } )}\right.$ & DSB $\left(\mathbf{M b p}^{-1} \mathbf{k G y}^{-1}\right)$ \\
\hline 100 & 0.2202 & $69.81 \pm 8.72$ & $3.66 \pm 0.43$ \\
\hline 150 & 0.2238 & $80.30 \pm 3.06$ & $3.71 \pm 0.11$ \\
\hline 200 & 0.2263 & $50.27 \pm 4.19$ & $3.83 \pm 0.45$ \\
\hline
\end{tabular}

Table 2. SSB and DSB yields following dry plasmid irradiation with $100-200 \mathrm{MeV}$ electrons calculated using the McMahon DNA damage $\mathrm{fit}^{32}$. Standard error based on three AGE repeats.

\begin{tabular}{|c|c|c|c|c|}
\hline \multirow[b]{3}{*}{ Energy $(\mathrm{MeV})$} & \multicolumn{4}{|l|}{ VHEE Dose rate } \\
\hline & \multicolumn{2}{|c|}{ Conventional $\left(\sim 0.5 \mathrm{~Gy} \mathrm{~s}^{-1}\right)$} & \multicolumn{2}{|c|}{ FLASH $\left(>10^{8} \mathrm{~Gy} \mathrm{~s}^{-1}\right)$} \\
\hline & SSB $\left(\mathrm{Mbp}^{-1} \mathrm{~Gy}^{-1}\right)$ & $\operatorname{DSB}\left(\mathrm{Mbp}^{-1} \mathrm{~Gy}^{-1}\right)$ & SSB $\left(M_{b} p^{-1} G^{-1}\right)$ & DSB $\left(\mathrm{Mbp}^{-1} \mathrm{~Gy}^{-1}\right)$ \\
\hline 100 & $15.42 \pm 0.86$ & $0.35 \pm 0.02$ & $20.31 \pm 1.20$ & $0.37 \pm 0.03$ \\
\hline 150 & $17.63 \pm 0.57$ & $0.35 \pm 0.03$ & $18.74 \pm 0.52$ & $0.37 \pm 0.04$ \\
\hline 200 & $20.19 \pm 0.56$ & $0.38 \pm 0.02$ & $21.22 \pm 0.38$ & $0.38 \pm 0.02$ \\
\hline
\end{tabular}

Table 3. Single- and double-strand break yields following aqueous plasmid irradiation with $100-200 \mathrm{MeV}$ electrons at Conventional and FLASH dose rates, calculated using the $\mathrm{McMahon}^{32}$ fit. Standard error based on six agarose gel electrophoresis repeats for $200 \mathrm{MeV}$ and five for 150 and $100 \mathrm{MeV}$.

Wet sample irradiations. Break yields were compared between plasmid DNA irradiated at a low dose rate $(\sim 0.5 \mathrm{~Gy} / \mathrm{s})$ and at FLASH dose rate $\left(>10^{8} \mathrm{~Gy} / \mathrm{s}\right)$. As discussed in the Introduction, significant differences in DSB yield were not anticipated due to the experimental conditions lacking key features understood to result in a FLASH effect. The plasmid was diluted as appropriate and samples placed in Eppendorf tubes. The SSB and DSB yields based on the McMahon fit are shown in Table 3 and Fig. 2.

To determine the contribution of direct and indirect effects to overall DNA damage, damage yields for dry and aqueous plasmid samples irradiated at Conventional dose rate were compared, with dry yields assumed to be caused by direct effects only and aqueous yields by both direct and indirect effects. The data is shown in Table 4, indicating that, as for protons and photons ${ }^{31}$, indirect effects from VHEE cause $>99 \%$ of damage in aqueous solutions with low scavenging capacity.

RBE calculation. DSB yields from dry and aqueous plasmid irradiations were used as the endpoint for calculating VHEE $\mathrm{RBE}^{33}$, referred to from this point as $\mathrm{RBE}_{\mathrm{DSB}}$ :

$$
R B E_{D S B}=\phi_{e^{-}} / \phi_{\gamma}
$$

where $\phi_{e^{-}}$is the DSB yield following plasmid irradiation by electrons and $\phi_{\gamma}$ is the DSB yield following irradiation by ${ }^{60} \mathrm{Co} \mathrm{X}$-rays - measured as $3.27 \pm 0.13 \mathrm{Mbp}^{-1} \mathrm{kGy}^{-1}$ for dry and $0.32 \pm 0.02 \mathrm{Mbp}^{-1} \mathrm{~Gy}^{-1}$ for wet plasmids based on experiments performed at the Dalton Nuclear Facility $(\mathrm{UK})^{34}$. $\mathrm{RBE}_{\mathrm{DSB}}$ values over the energy range 100-200 MeV are given in Table 5 below, along with values for other radiotherapy modalities ${ }^{35-38}$ in Fig. 3 and Table 5. 

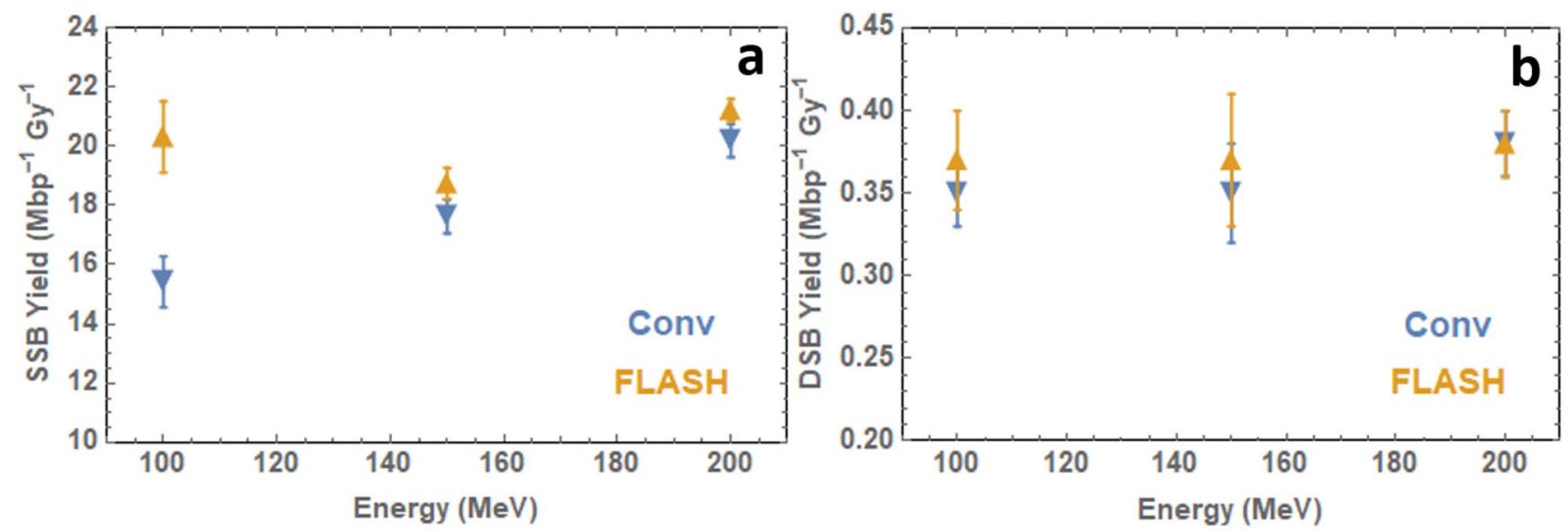

Figure 2. (a) Single-strand break yields and (b) double-strand break yields for 100-200 MeV electron beam irradiation of wet pBR322 plasmid DNA based on data fitting to the McMahon fit $^{32}$. Plasmids irradiated at Conventional $(\sim 0.5 \mathrm{~Gy} / \mathrm{s})$ and FLASH $\left(>10^{8} \mathrm{~Gy} / \mathrm{s}\right)$ dose rates.

\begin{tabular}{|l|l|l|}
\hline Energy $(\mathrm{MeV})$ & \% of SSBs from indirect damage & \% of DSBs from indirect damage \\
\hline 100 & $99.54 \pm 0.03$ & $99.0 \pm 0.05$ \\
\hline 150 & $99.54 \pm 0.01$ & $98.9 \pm 0.07$ \\
\hline 200 & $99.75 \pm 0.01$ & $99.0 \pm 0.05$ \\
\hline
\end{tabular}

Table 4. Percentage of total DNA damage caused by indirect radiation effects for SSBs and DSBs.

\begin{tabular}{|c|c|c|c|}
\hline Particle & Energy (MeV) & RBE & Biological Endpoint \\
\hline \multirow{6}{*}{$\mathrm{e}^{-}$(this work, CLEAR) } & \multirow{2}{*}{100} & $1.12 \pm 0.13$ & Dry plasmid DSB yield \\
\hline & & $1.09 \pm 0.09$ & Wet plasmid DSB yield \\
\hline & \multirow{2}{*}{150} & $1.13 \pm 0.06$ & Dry plasmid DSB yield \\
\hline & & $1.09 \pm 0.12$ & Wet plasmid DSB yield \\
\hline & \multirow{2}{*}{200} & $1.17 \pm 0.14$ & Dry plasmid DSB yield \\
\hline & & $1.19 \pm 0.10$ & Wet plasmid DSB yield \\
\hline \multirow{3}{*}{$\mathrm{e}^{-}($Small $)$} & 6 & $0.97 \pm 0.11$ & Wet plasmid DSB yield \\
\hline & 10 & $0.94 \pm 0.07$ & Wet plasmid DSB yield \\
\hline & 15 & $0.91 \pm 0.06$ & Wet plasmid DSB yield \\
\hline \multirow{2}{*}{$\mathrm{e}^{-}$(Herskind) } & \multirow{2}{*}{10} & $0.94 \pm 0.02$ & V79 survival fraction $=0.0003$ (rel. to $6 \mathrm{MV} \mathrm{X-rays)}$ \\
\hline & & $0.98 \pm 0.01$ & MCF7 survival fraction $=0.0003$ (rel. to $6 \mathrm{MV} \mathrm{X}$-rays) \\
\hline \multirow{4}{*}{$\mathrm{e}^{-}$(Spadinger) } & \multirow{4}{*}{11} & $1.1 \pm 0.08$ & V79 survival fraction of 0.1 ( $0-10 \mathrm{~Gy})$ \\
\hline & & $1.0 \pm 0.04$ & CHO survival fraction of $0.1(0-10 \mathrm{~Gy})$ \\
\hline & & $1.0 \pm 0.06$ & V79 survival fraction of 0.1 (0-3 Gy) \\
\hline & & $0.9 \pm 0.1$ & CHO survival fraction of 0.1 ( $0-3 \mathrm{~Gy})$ \\
\hline \multirow{2}{*}{$\mathrm{e}^{-}$(Zackinsson) } & \multirow{2}{*}{50} & $1.03 \pm 0.08$ & V79 survival fraction of 0.1 (rel. to 4 MV X-rays) \\
\hline & & $1.02 \pm 0.07$ & V79 survival fraction of 0.01 (rel. to 4 MV X-rays) \\
\hline \multirow{5}{*}{$\mathrm{p}($ Vysin $)$} & 10 & $1.4 \pm 0.62$ & Dry plasmid DSB yield \\
\hline & \multirow{2}{*}{20} & $1.00 \pm 0.41$ & Wet plasmid DSB yield \\
\hline & & $0.6 \pm 0.51$ & Dry plasmid DSB yield \\
\hline & \multirow{2}{*}{30} & $0.75 \pm 0.5$ & Wet plasmid DSB yield \\
\hline & & $0.5 \pm 0.25$ & Dry plasmid DSB yield \\
\hline \multirow{4}{*}{ X-rays (Zackinsson) } & \multirow{2}{*}{20} & $0.99 \pm 0.07$ & V79 survival fraction of 0.1 (rel. to 4 MV X-rays) \\
\hline & & $1.00 \pm 0.05$ & V79 survival fraction of 0.01 (rel. to 4 MV X-rays) \\
\hline & \multirow{2}{*}{50} & $1.14 \pm 0.07$ & V79 survival fraction of 0.1 (rel. to 4 MV X-rays) \\
\hline & & $1.12 \pm 0.05$ & V79 survival fraction of 0.01 (rel. to 4 MV X-rays) \\
\hline
\end{tabular}

Table 5. RBE for various particle modalities with particle, energy and biological endpoint specified. Standard error of the mean for Small data calculated based on gel repeats. RBE data taken or calculated from references ${ }^{27-31}$. All RBE calculations relative to ${ }^{60} \mathrm{Co} \mathrm{X}$-ray data unless specified. 

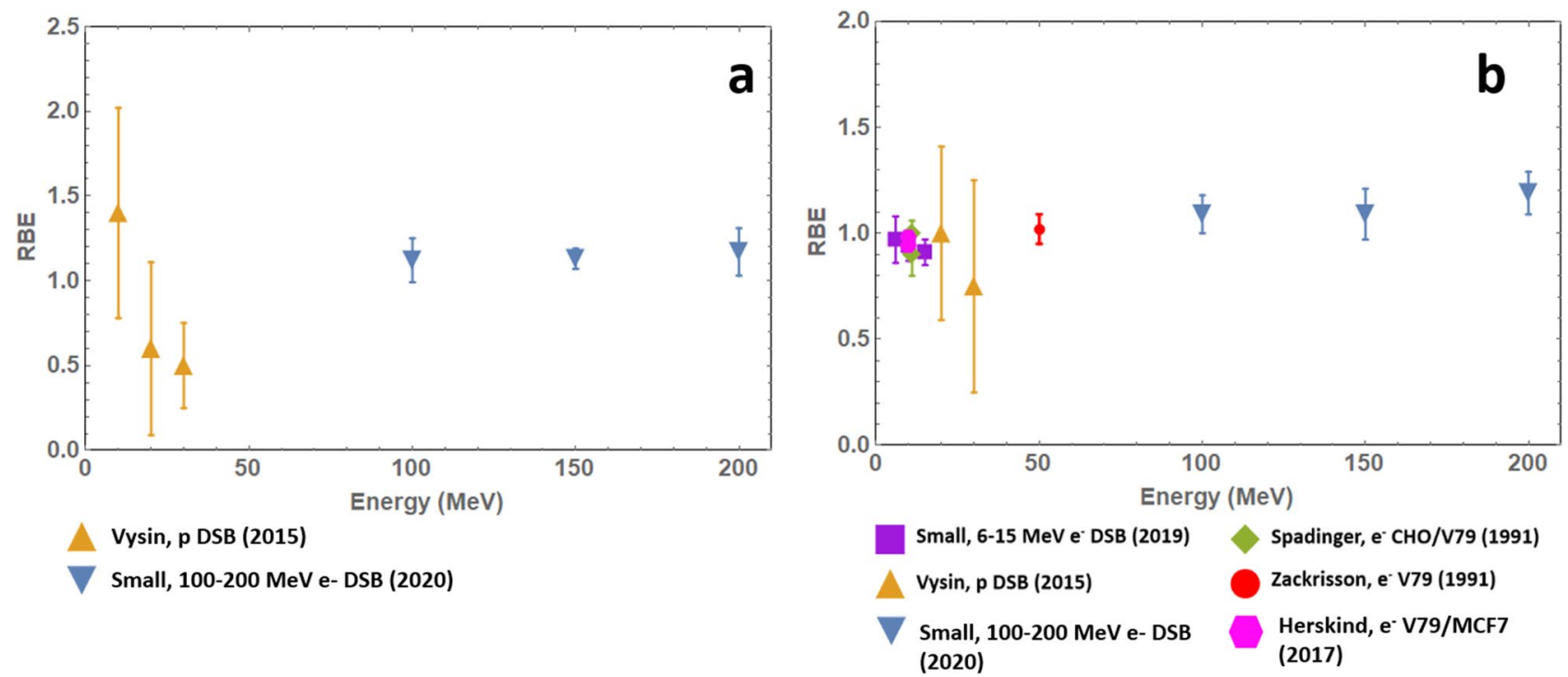

Figure 3. RBE values for (a) VHEE and protons with dry plasmid DSB yield as the biological endpoint and (b) VHEE, low-energy electrons and protons with wet plasmid DSB yield (Small, Vysin, CLEAR) or cell survival fraction (Herskind, Spadinger, Zackrisson) as the biological endpoint. Experimental data taken from references $^{35-38}$.

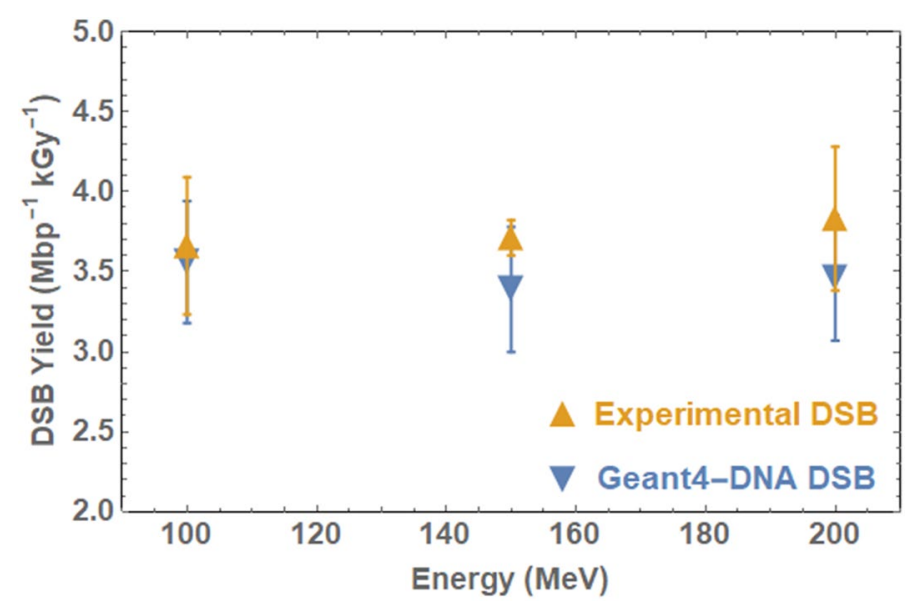

Figure 4. Double-strand break yields for experimental and computational plasmid DNA irradiation with 100-200 MeV electrons. Geant4-DNA simulation performed with half-cylinder DNA geometry with a $10 \mathrm{bp}$ separation defined for DSB induction and damage determined by an energy threshold of $8.22 \mathrm{eV}$. Standard errors calculated based on $10^{3}$ repeats.

Comparison of GEANT4-DNA and experimental DSB yields. Experimental DSB data was then compared to GEANT4-DNA simulation data, modelling dry plasmid irradiation with VHEE beams at the same energies as those used at CLEAR. DSB data was chosen as experimental SSB data is likely to be less reliable due to transportation effects. As the parameters based on Henthorn's earlier work on protons resulted in a significant underestimate in DSB yield compared with experimental data for electrons, a parameter variation study was performed. The geometry, damage scoring method and base pair separation were varied according to similar studies on DNA damage modelling ${ }^{39-41}$ to determine the optimal conditions for simulating plasmid damage with GEANT4-DNA following electron irradiation. This study indicated that use of the energy threshold damage mechanism at $8.22 \mathrm{eV}$ with a separation of $10 \mathrm{bp}$ for DSB induction results in DSB yields which most closely approximated experimental data (Fig. 4), with a more complete set of results available in the Supplementary Material (SF1-SF3).

\section{Discussion}

This work presents the results of the first plasmid irradiation experiments using VHEE at the CLEAR user facility with the aims of investigating the physical and chemical damage caused to DNA following VHEE irradiation. pBR322 plasmids were irradiated in both dry and aqueous environments to investigate the contributions from 
direct and indirect radiation effects arising from the production of hydroxyl and other radicals. The effect of dose-rate variation was also studied at the DNA damage level, following the rapid resurgence of FLASH radiotherapy and the potential suitability of VHEE as a FLASH radiotherapy modality. DSB yields were then used as the biological endpoint to calculate VHEE RBE.

pBR322 plasmids irradiated in dry and aqueous environments showed little variation in DSB induction over $100-200 \mathrm{MeV}$, likely due to there being correspondingly little variation in LET $(0.220-0.226 \mathrm{keV} / \mu \mathrm{m})$. Significant variation was observed in the dry SSB yield with electron energy. Relaxing of the supercoiled form of the plasmid to the open circular form was found to have occurred during transportation, resulting in a higher proportion of the plasmid being in an open-circular state before irradiation. For future experiments, it would be recommended that the pBR322 plasmid be shipped directly from New England BioLabs to the facility in the buffer to prevent relaxation and ensure the reliability of SSB measurement.

Comparison of the break yields in aqueous and dry environments allowed the contribution of indirect radiation effects to induced DNA damage to be measured. As anticipated, SSB and DSB yields indicate that indirect effects are the primary cause of DNA damage, causing in excess of $99 \%$ of damages. While this seems to contradict the conclusions of Ward et $\mathrm{al}^{42}$. that indirect effects contribute to $\sim 65 \%$ of total radiation effects, it is important to note that Ward's conclusion is based on irradiation of cells, which typically have a scavenging capacity of approximately 200 times that of the diluted aqueous plasmid solution in this study ( $1 \mathrm{mM}$ Tris). As more radicals are able to cause DNA damage in this plasmid solution, a significantly higher proportion of indirect damage is therefore measured. These results are consistent with Vysin's measurements of the direct and indirect effect contributions to proton damage of plasmids, but are not directly translatable to cells or tissues due to the significantly lower scavenging capacity.

The effect of dose rate on the irradiation of aqueous plasmids was also investigated, with the aim to determine whether a 'FLASH' effect could be observed at the nanoscopic level, in the form of variation in DSB yields following irradiation at conventional or ultra-high dose rates. Figure $2 \mathrm{~b}$ indicates that there is no statistically significant variation in DSB yield with dose rate. This result is in agreement with our hypothesis that significant DSB variation would not be observed between plasmids irradiated by VHEE at conventional and ultra-high dose rates due to the lack of key experimental features which are the cause of the FLASH effect, notably the use of oxygenated purified water, and room temperature $\left(25^{\circ} \mathrm{C}\right)$ environment. This conclusion is similar to those drawn by other ultra-high dose rate radiobiological studies, though this is understood to be the first study of its kind involving ultra-high dose rate irradiation of plasmid DNA with VHEE. As VHEE is an increasingly popular potential modality for FLASH therapy, a fundamental understanding of the physical effects of FLASH irradiation is crucial.

DSB yields from the dry plasmid irradiation and conventional wet plasmid irradiation experiments were used as the biological endpoint for RBE calculation and compared with RBE of other radiotherapy modalities. VHEE $\mathrm{RBE}_{\mathrm{DSB}}$ was found to be close to 1 for dry plasmids and 1.1-1.2 for wet plasmids. Comparison with values for clinical electrons suggests that electron RBE may increase with energy.

GEANT4-DNA simulations were carried out in parallel, modelling dry plasmid irradiation with 100-200 MeV electrons to determine the model parameters which could best approximate the experimental conditions. The results of plasmid irradiation simulations indicate that a DNA model built using half-cylinder geometry with damage scored using an energy threshold of $8.22 \mathrm{eV}$ and in which DSBs are induced when two SSBs occur within a separation of 10 base pairs, most closely approximates the CLEAR data. The parameters used here are similar, with small differences, compared to previous in silico studies based on literature-reported experimental data using other radiation modalities including protons, albeit with large variation across datasets. Small differences are likely due to differences between experimental and in silico configuration.

In conclusion, a set of plasmid irradiation experiments was successfully performed at the CLEAR user facility using 100-200 MeV electrons. Little DSB yield variation was observed over the energy (and therefore LET) range. Indirect effects were calculated to contribute $>99 \%$ of observed plasmid breaks, consistent with observations for other modalities. No significant variation in damage yield was observed with dose-rate variation, indicating that a FLASH effect is not present, at least for VHEE, at the nanoscale within the plasmid irradiations. Finally, it has been shown, through $\mathrm{RBE}_{\mathrm{DSB}}$ calculations, that the physical damage caused to DNA by VHEE is similar to that caused by ${ }^{60} \mathrm{Co} \mathrm{X}$-rays and low-energy electrons. This provides an indication that more complex biological effects such as cell death could also be similar. This is a key initial pre-clinical step on the way to clinical implementation of VHEE radiotherapy.

\section{Methods}

Plasmid sample setup. pBR322 plasmid DNA (New England Biolabs) isolated from E.Coli (4361 base pairs $)^{30}$ was used in this study. This cloning vector has been extensively used as a plasmid model system in irradiation studies irradiation studies ${ }^{38,43,44}$, allowing direct comparison to be made between this and earlier studies. The plasmid, in solution containing $10 \mathrm{mM}$ Tris- $\mathrm{HCl}$ and $1 \mathrm{mM}$ EDTA buffers to prevent degradation during freeze-thaw cycles, was diluted with purified water from $1000 \mathrm{ng} / \mu \mathrm{l}$ to $100 \mathrm{ng} / \mu \mathrm{l}$. New England BioLabs quotes that $\sim 90 \%$ of the plasmid is in a supercoiled (undamaged) form. Agarose gel electrophoresis confirmed that between 85 and $90 \%$ of the unirradiated plasmid was in this form.

Dry samples were prepared by pipetting $5 \mu \mathrm{l}$ droplets of $100 \mathrm{ng} / \mu \mathrm{l}$ plasmid DNA directly on to the centre of Permafrost glass microscope slides $\left(25 \times 75 \times 1 \mathrm{~mm}^{3}\right.$, Thermo Fisher Scientific). The droplets were left to dry at room temperature, leaving a thin layer of DNA on the slide. Aqueous samples were held in sealed $1.5 \mathrm{ml}$ Eppendorf tubes, with each tube containing $30 \mu \mathrm{l}$ of plasmid solution at $100 \mathrm{ng} / \mu \mathrm{l}$. All samples were stored at $-20^{\circ} \mathrm{C}$ before and after irradiation. 

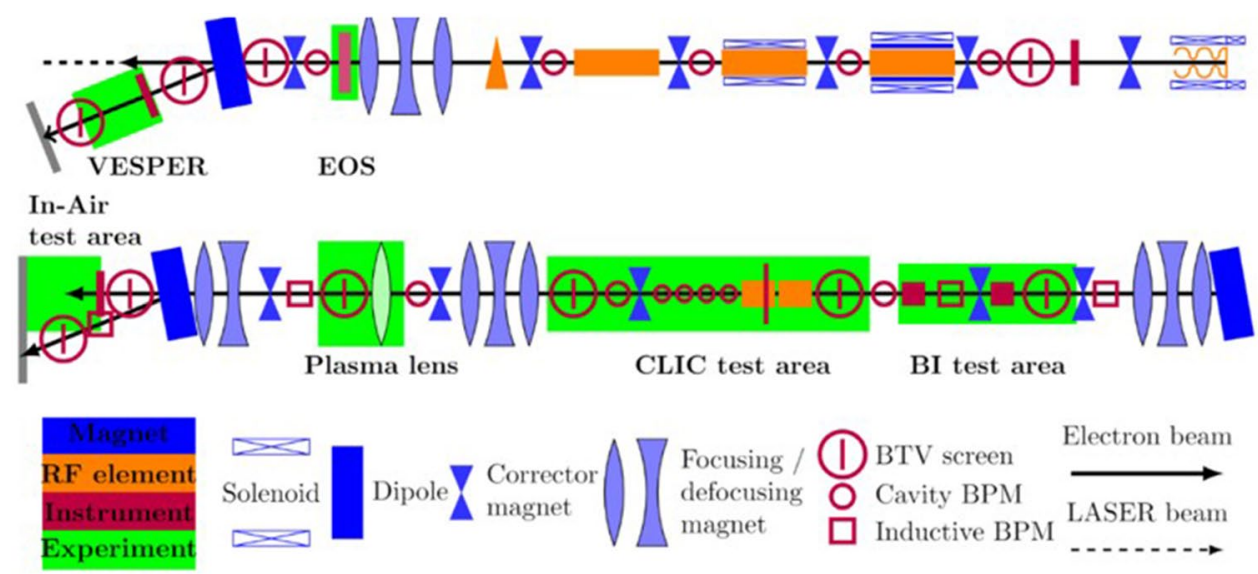

Figure 5. Schematic of the CLEAR beamline and two experimental areas, Figure produced by Kyrre Sjobak ${ }^{22}$ and reproduced here with kind permission from Kyrre Sjobak.

\begin{tabular}{|l|l|}
\hline Parameter & Value \\
\hline Beam energy & $60-220 \mathrm{MeV}$ \\
\hline Bunch charge & $0.001-10 \mathrm{nC}$ \\
\hline No. bunches & Variable: $0->200$ \\
\hline Beam repetition rate & $0.833-10 \mathrm{~Hz}$ \\
\hline \multirow{2}{*}{ Bunch repetition rate } & $1.5 \mathrm{GHz}$ (high bunch charge) \\
\cline { 2 - 2 } & $3 \mathrm{GHz}$ (low bunch charge) \\
\hline RMS energy spread & $<0.2 \%$ \\
\hline Typical dose per shot & $\begin{array}{l}34.29 \mathrm{~Gy} / \text { shot }(200 \mathrm{MeV}, \text { dry) } \\
0.69 \mathrm{~Gy} / \text { shot }(200 \mathrm{MeV}, \text { wet, Conv) }\end{array}$ \\
\hline
\end{tabular}

Table 6. CLEAR beam parameters primarily taken from Gamba et al. ${ }^{21}$ and Sjobak et al. ${ }^{22}$ Beam charge and repetition rate updated based on current availability.

Irradiation setup. Plasmid irradiations were carried out at the CLEAR user facility (CERN, Geneva), an $\mathrm{S}$-band linear accelerator designed primarily for research and development applications $\mathrm{s}^{21,22}$. CLEAR is housed in the previous CLEX experimental area and consists of the $25 \mathrm{~m}$ CALIFES (Concept d'Accélérateur Linéare pour Faisceau d'Electron Sonde) injector, adapted from previous use to test and prove the feasibility of novel two-beam accelerator technology, and a $16 \mathrm{~m}$ user beamline. The beamline has two experimental areas, with the in-air beam end area selected for this experiment (see Fig. 5).

The CLEAR user facility was chosen for VHEE plasmid irradiation experiments as it can produce stable highenergy electron beams over an energy range of 60-220 MeV, with readily adaptable beam size, bunch charge and energy-see Table 6 for a full description of beam parameters. CLEAR also has a strong history of VHEE experiments over recent years, including inhomogeneity sensitivity and dosimetry studies from the University of Manchester ${ }^{6,45,46}$.

The experiment was built in the in-air test area, a $1 \mathrm{~m}$ space beyond the exit window through which the beam travels before reaching a concrete beam dump. Beam energy and bunch charge were measured using a dipole spectrometer and a Bergoz Integrated Current Transformer respectively. A YAG screen, placed approximately $2 \mathrm{~cm}$ behind the plasmid samples measured the beam size, shape and position. Lead bricks provided shielding from secondary X-rays, with a small opening allowing the beam to reach the samples.

Dry samples were slotted into a 3D-printed polylactic acid (PLA) slide holder, custom-designed and built at the Cockcroft Institute. This was mounted on to a transversely moving stage placed in front of the beam, presenting each sample to the beam in turn. Dry samples were irradiated at 100, 150 and $200 \mathrm{MeV}$ over a dose range of 1000-6000 Gy with 3 repeats made for each energy and each dose. A schematic and image of the dry experiment setup is shown in Fig. 6.

Aqueous samples were held in $1.5 \mathrm{ml}$ sealed Eppendorf tubes and mounted in an aluminium tube holder (see Fig. 7), with irradiation carried out in a similar manner to the dry samples. The samples were again irradiated at 100,150 and $200 \mathrm{MeV}$, over a dose range of 0-50 Gy. The difference in dose between dry and aqueous samples arises due to the contribution of direct and indirect effects. To generate observable levels of damage, dry samples must receive a significantly higher dose.

To determine the charge required to deliver the required dose, simulations of the dry and aqueous experimental setups were performed in TOPAS. The dose delivered by $10^{7}$ electrons was recorded, scaled up to the required 

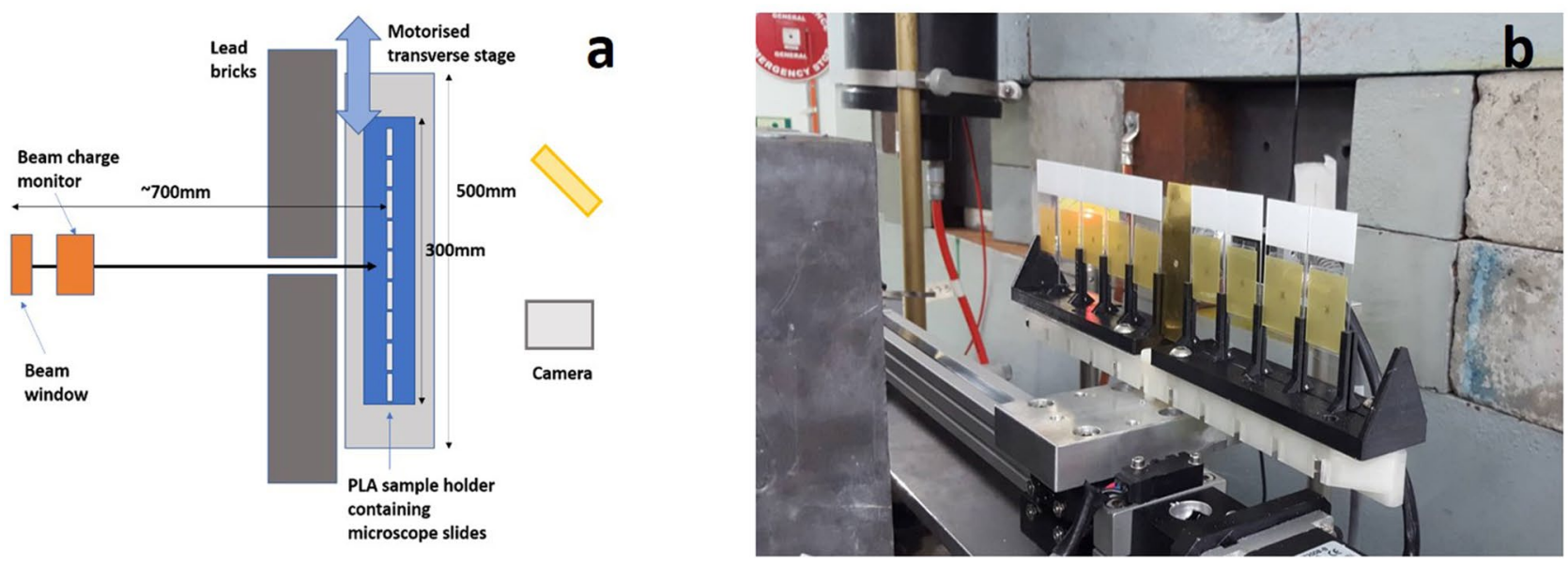

Figure 6. (a) Schematic (produced by author K.L.S.) and (b) photograph (taken by author K.L.S.) of experimental set-up for irradiation of dry plasmid samples on glass microscope slides. EBT-XD film placed behind samples to show sample coverage by beam.
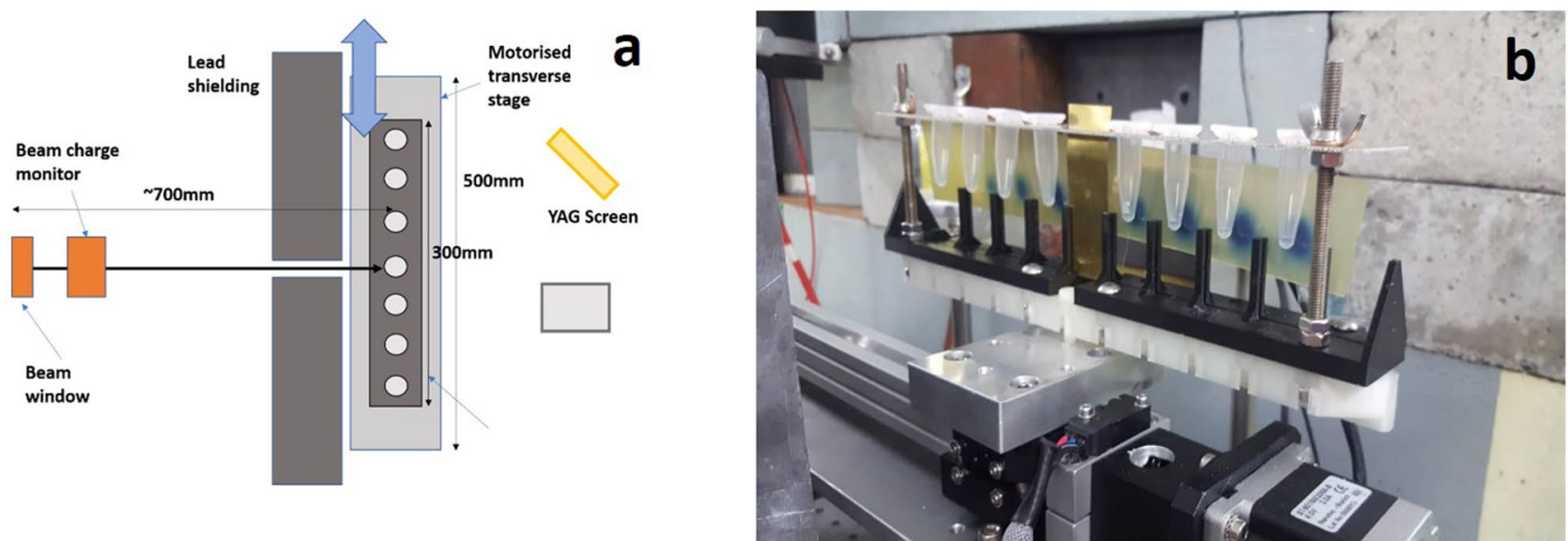

Figure 7. (a) Schematic (produced by author K.L.S. and (b) photograph (taken by author K.L.S.) of experimental set-up for irradiation of wet plasmid samples in Eppendorf tubes. EBT-XD film placed behind samples to determine dose delivered to samples.

dose and the corresponding charge determined. This method has been verified to within a maximum error of $5.26 \%$ by Lagzda et al. ${ }^{6,45,46}$. For both dry and wet irradiations, EBT-XD film, a radiochromic film commonly used in radiotherapy dosimetry with a dynamic range of $0.1-60 \mathrm{~Gy}^{47}$, was placed behind the samples. For the aqueous samples, the film was used to determine the dose delivered to the film, based on $15 \mathrm{MeV}$ electron beam calibration performed at the Christie NHS Foundation Trust using a Varian treatment linac. For the dry samples the film was used to confirm sample coverage only as the dynamic dose range of the film (0-60 Gy) is too low, with dose instead determined from the beam fluence, with beam $\sigma$ measured using a YAG screen and charge from the beam measured using an ICT. TOPAS simulations revealed a 13-14\% difference in dose across the glass slide, which was accounted for in final dose calculations. Further detail is given in the Supplementary material (SF4).

The effect of conventional and FLASH-level dose rates was investigated for aqueous samples to determine if a FLASH effect could be observed at the DNA damage level. The FLASH dose rates were reached by increasing the bunch charge and delivering the radiation in ultra-short $(\sim \mathrm{ps})$ single pulses, resulting in dose rates calculated using individual bunch duration in excess of $10^{8} \mathrm{~Gy} / \mathrm{s}$.

Agarose gel electrophoresis (AGE). Unirradiated plasmid DNA exists in an undamaged, or supercoiled (SC), state. Ionising radiation causes SSBs and DSBs within the DNA, detectable as a change in the plasmid form. Open-circular (OC) plasmid results from a SSB, due to relaxation of the SC DNA. DSBs are detectable as linear (L) forms of plasmid ${ }^{14}$. Determination of plasmid forms was assessed through AGE.

Dry samples were recovered from the glass slides using $5 \mu \mathrm{l}$ of purified water and the $30 \mu \mathrm{l}$ aqueous samples were split into six $5 \mu \mathrm{l}$ sub-samples. Each sample was mixed with $1 \mu \mathrm{l}$ gel loading dye and loaded into $5 \mathrm{~mm}$ wells in a $1 \% \mathrm{w} / \mathrm{v}$ agarose gel in $1 \times \mathrm{TAE}$ buffer stained with $20 \mu \mathrm{l}$ SYBR Green. The gel was submerged in $0.5 \times \mathrm{TAE}$ buffer and a $100 \mathrm{~V}$ voltage applied. The gel was run for $120 \mathrm{~min}$ or until the samples had migrated $70-80 \%$ through the gel. The gels were imaged using a ChemiDoc MP UV imager (BioRad). The plasmid forms appear 

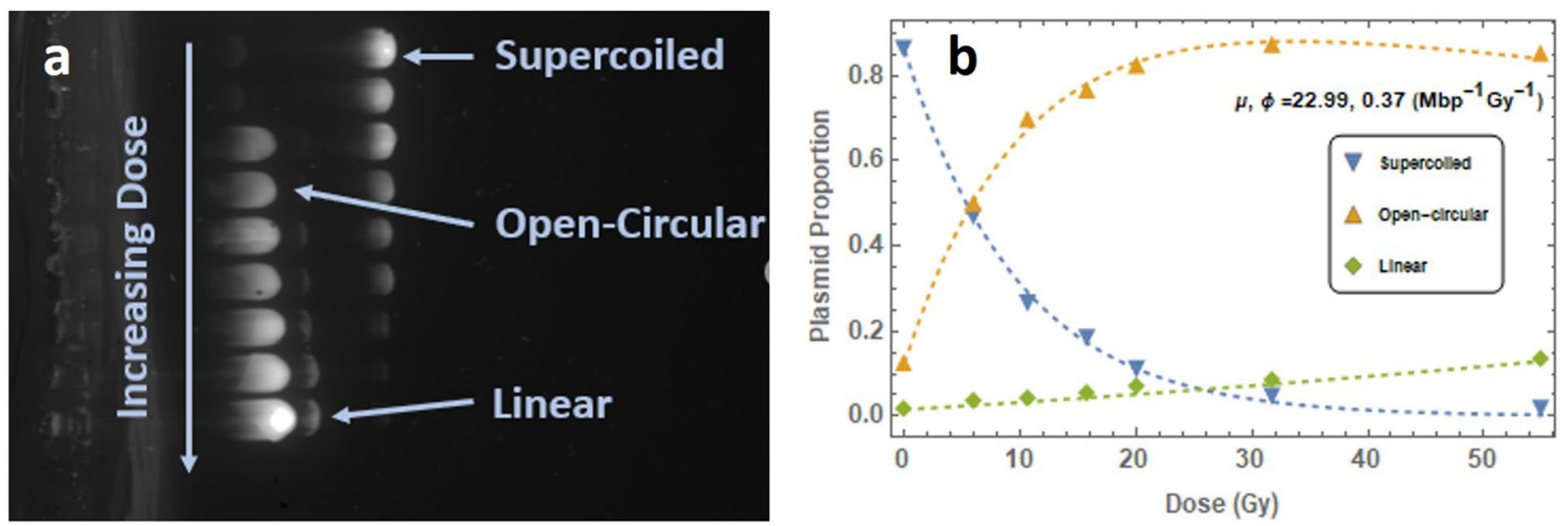

Figure 8. (a) Cropped UV image of agarose gel following electrophoresis containing wet plasmids irradiated by $100 \mathrm{MeV}$ electrons. Labelled bands indicate presence of SC, OC and L plasmid forms. Uncropped image shown in SF5. (b) SC, OC and L plasmid proportions as a function of dose following integration of band intensities, fitted to Eqs. (2), (3) and (4). Error bars calculated based on five gel repeats.

as distinct bands along the gel (Fig. 8a). Fiji ${ }^{48}$, an open-source image processing package based on ImageJ was used to determine the relative intensities of these bands for each sample, normalised with respect to the most intense band. The proportion of each plasmid form was calculated by integrating the signal over each band.

Modelling plasmid damage. While several potential fitting methods ${ }^{49}$ are available to determine the yield of SSB and DSBs on DNA, the McMahon ${ }^{32}$ fit was selected for this study based on it being specifically developed for fitting plasmid form proportion data obtained from AGE after irradiation and following a study by Vysin ${ }^{31}$ on the robustness of different fits. The plasmid data are fitted to the following equations:

$$
\begin{gathered}
S C(D)=S_{0} e^{-(\mu D+\phi D)} \\
O C(D)=e^{-\phi D}\left[e^{-\frac{1}{2} \mu^{2} \rho D^{2}}\left(S_{0}+C_{0}\right)-S_{0} e^{-\mu D}\right] \\
L(D)=1-\left(S_{0}+C_{0}\right) e^{-\left(\phi D+\frac{1}{2} \mu^{2} \rho D^{2}\right)}
\end{gathered}
$$

where $S C(D), O C(D)$ and $L(D)$ are the proportion of supercoiled, open-circular and linear plasmid respectively after irradiation of dose $D$ in Gy, $\mu$ and $\phi$ are the average SSB and DSB yields $\left(\mathrm{Mbp}^{-1} \mathrm{~Gy}^{-1}\right), S_{0}$ and $C_{0}$ are the supercoiled and open-circular proportions at zero dose and $\rho$ is the probability of a DSB arising due to two SSBs on opposite DNA strands within 10 base pairs. pBR322 plasmid consists of 4361 base pairs, giving $\rho=10 / 4361$.

Figure $8 \mathrm{~b}$ shows the proportions of SC, OC and L plasmid forms as a function of dose. A least-square error non-linear fit was made to the SC and OC equations to obtain the SSB and DSB yields ( $\mu$ and $\varphi$ resepectively). The agreement between the L equation and the linear plasmid data, as observed in Fig. $8 \mathrm{~b}$, indicates the efficacy of the McMahon fit.

Plasmid irradiation simulations with GEANT4-DNA. Plasmid irradiation simulations were carried out to compare DNA damage yields with those obtained from experimental studies. The simulations were carried out using GEANT4-DNA, a module based on the Monte-Carlo particle tracking code GEANT4 (version 10.02-patch01 $)^{50}$ designed to model biological damage induced by ionising radiation at the DNA scale ${ }^{51-54}$.

The simulation is based on a plasmid irradiation model designed originally for interaction of proton beams with DNA by Henthorn et al. ${ }^{55}$ pBR322 plasmid DNA, consisting of 4361 base pairs and with radius $236 \mathrm{~nm}$, was built and interaction with monoenergetic electron beams simulated. The GEANT4-DNA default physics list is capable of simulating electrons up to $1 \mathrm{MeV}$ so, to allow use with high energy electrons, the Livermore physics list (G4EmLivermorePhysics) for electrons with energy $>1 \mathrm{MeV}$ was added ${ }^{56}$, with the energy range over which the model functions set to $1-300 \mathrm{MeV}$.

The irradiation model consists of a circular plasmid placed on a glass slab of density $2.23 \mathrm{~g} / \mathrm{cm}^{3}$, representing the microscope slide, held within air. The plasmid DNA is built based on a half-cylindrical geometry, as proposed by Charlton et al. ${ }^{57}$. As a simplification, the plasmid geometry is modelled as a closed circle, similar to the approach of McNamara et al. ${ }^{58}$ with the simplified DNA geometry (half-cylinder) as published by Bernal ${ }^{59}$. Each discrete half cylinder is numbered to determine the base pair position on the plasmid. The electron beam is directed perpendicular to the plasmid. Dose to the dry samples was calculated based on the beam fluence, determined using CLEAR beam diagnostics. As the simulation consisted of a single plasmid, the beam radius was scaled down to $300 \mathrm{~nm}$. To ensure the same beam fluence as in the experiment, the number of particles $\mathrm{N}$ required to deliver the correct dose was calculated using the following: 


$$
N=\frac{\pi D \rho r^{2}}{10^{9} e L}
$$

where $\mathrm{L}$ is the electron LET in units of $\mathrm{keV} / \mu \mathrm{m}, \mathrm{D}$ is the radiation dose in Gray, $\mathrm{r}$ is the beam radius in $\mathrm{m}$ and $\rho$ is the DNA density, set at $1407 \mathrm{~kg} \mathrm{~m}^{-3}$.

DNA damage is scored using the same approach as Henthorn ${ }^{47}$ of using two different energy deposition mechanisms to determine the sensitivity of DNA damage yield with energy deposition. The first is based on energy deposition corresponding to a damage probability which increases linearly from 0 to 1 over the energy range $5-37.5 \mathrm{eV}$, informed by photon and low energy electron DNA damage studies ${ }^{59}$. The second is based on an energy threshold-an energy deposition over this threshold is considered to have caused damage to the DNA. A commonly used value is $17.5 \mathrm{eV}^{40}$, though a review of several studies has shown a range of $8.22-22.5 \mathrm{eV}$ in use $\mathrm{e}^{41}$.

The damages scored on the DNA volumes are defined as SSBs. DSBs are determined through a clustering algorithm searching for SSBs which have occurred on opposite DNA strands within a specified distance. Output files show the damage data as the number of damages occurring in a single run. The average DSB yields over $10^{3}$ runs are reported per Mbp per kGy.

Received: 6 August 2020; Accepted: 7 December 2020

Published online: 08 February 2021

\section{References}

1. National Cancer Registration \& Analysis Service and Cancer Research UK. Chemotherapy, Radiotherapy and Tumour Resections in England: 2013-2014 Workbook. http://www.ncin.org.uk/cancer_type_and_topic_specific_work/topic_specific_work/ main_cancer_treatments (2017)

2. NHS. (2020). Proton beam therapy. Available: https://www.england.nhs.uk/commissioning/spec-services/highly-spec-services/ $\mathrm{pbt} /$. Last accessed 28th Jul 2020.

3. Jones, R. M. Wakefield suppression in high gradient linacs for lepton linear colliders. Phys. Rev. ST Accel. Beams 12, 104801 (2009).

4. Jones, R. M. et al. Influence of fabrication errors on wake function suppression in NC X-band accelerating structures for linear colliders. New J. Phys. 11, 033013 (2009).

5. DesRosiers, C., Moskvin, V., Bielajew, A. F. \& Papiez, L. 150-250 MeV electron beams in radiation therapy. Phys. Med. Biol. 45, 1781-1805 (2000).

6. Lagzda, A. et al. Very-high energy electron (VHEE) studies at CERN'S CLEAR user facility. Proc. 9th Int. Particle Accelerator Conf. (2018)

7. Bazalova-Carter, M. et al. Treatment planning for radiotherapy with very high-energy electron beams and comparison of VHEE and VMAT plans. Med. Phys. 42, 2615-2625 (2015).

8. Vozenin, M. C., Hendry, J. H. \& Limoli, C. L. Biological benefits of ultra-high dose rate FLASH radiotherapy: sleeping Beauty awoken. Clin. Oncol. 31, 407-415 (2019).

9. Favaudon, V. et al. Ultrahigh dose-rate FLASH irradiation increases the differential response between normal and tumour tissue in mice. Sci. Transl. Med. 6, 1-9 (2014).

10. Monte-Gruel, P. Irradiation in a FLASH: Unique sparing of memory in mice after whole-brain irradiation with dose rates above 100 Gy/s. Radiother. Oncol. 124, 365-369 (2017).

11. Vozenin, M. C. et al. The advantage of FLASH radiotherapy confirmed in mini-pig and cat-cancer patients. Clin. Cancer Res. 25, 35-42 (2018).

12. Bourhis, J. et al. Treatment of a first patient with FLASH-radiotherapy. Radiother. Oncol. 139, 18-22 (2019).

13. Maxim, P. G., Tantawi, S. G. \& Loo, B. W. PHASER: A platform for clinical translation of FLASH cancer radiotherapy. Radiother. Oncol. 139, 28-33 (2019).

14. Scholes, G., Ward, J. F. \& Weiss, J. Mechanism of the radiation-induced degradation of nucleic acids. J. Mol. Biol. 2, 379-391 (1960).

15. Suntharalingam, N., Podgorsak, E. B., Hendry, J. H. (2005). 14. Basic Radiobiology. In: Radiation Oncology Physics: A Handbook for Teachers and Students. (Vienna: International Atomic Energy Agency) 485-504

16. Chang, D. S., Lasley, F. D., Das, I. J., Mendonca, M. S., Dynlacht, J. R. Molecular mechanisms of DNA damage and repair in: Basic radiotherapy physics and biology 201-208 (Springer, 2014)

17. Eriksson, D. \& Stigbrand, T. Radiation-induced cell death mechanisms. Tumor Biol. 31, 363-372 (2010).

18. Nikjoo, H. \& Lindborg, L. RBE of low energy electrons and photons. Phys. Med. Biol. 55, 65-109 (2010).

19. Paganetti, H. Relative biological effectiveness (RBE) values for proton therapy. Variations as a function of biological endpoint, dose and linear energy transfer. Phys. Med. Biol. 59, R419-R472 (2014).

20. Thomas, C. M., Summers, D. Bacterial Plasmids in: Encyclopedia of Life Sciences (ELS). (Wiley, 2018)

21. Gamba, D. et al. The CLEAR user facility at CERN. Nucl. Inst. Methods Phys. Res. A 909, 480-483 (2018).

22. Sjobak, K. N. et al. Status of the CLEAR electron beam user facility at CERN. Proc. 10th Int. Particle Accelerator Conf. (2019)

23. Town, C. D. Effect of high dose rates on survival of mammalian cells. Nature 215, 847-848 (1967).

24. Beyreuther, E. et al. Radiobiological response to ultra-short pulsed megavoltage electron beams of ultra-high pulse dose rate. Int. J. Radiat. Biol. 91(8), 643-652 (2015).

25. Babayan, N. et al. Dose-rate effect of ultrashort electron beam radiation on DNA damage and repair in vitro. Radiat. Res. 58(6), 894-897 (2017).

26. Rigaud, O. et al. Exploring ultrashort high-energy electron-induced damage in human carcinoma cells. Cell Death Dis. 1, e73 (2010).

27. Yogo, A. et al. Application of laser-accelerated protons to the demonstration of DNA double-strand breaks in human cancer cells. Appl. Phys. Lett. 95, 181502 (2009).

28. Hanton, F. et al. DNA DSB repair dynamics following irradiation with laser-driven protons at ultra-high dose rates. Sci. Rep. 9, 4471 (2019).

29. Ozols, A., Prise, K. \& Michael, B. D. A comparison of the radiosensitivity of relaxed and supercoiled plasmid DNA. Int. J. Radiat. Biol. 75(1), 83-90 (1999).

30. pBR322 Vector. New England BioLabs. https://www.neb.uk.com/products/neb-catalogue/nucleic-acids-\%28cloning\%29/pbr32 2-vector (2020)

31. Bolivar, F. et al. Construction and characterization of new cloning vehicles II. A multipurpose cloning system. Gene 2, 95-113 (1977).

32. McMahon, S. J. \& Currell, F. J. A robust curve-fitting procedure for the analysis of plasmid DNA strand break data from gel electrophoresis. Radiat. Res. 175, 797-805 (2011). 
33. Ou, H. et al. Monte Carlo simulation of the relative biological effectiveness and DNA damage from a $400 \mathrm{MeV} / \mathrm{u}$ carbon ion beam in water. Appl. Radiat. Isot. 136, 1-92 (2018).

34. Small. K., et al. A comparative study of biological effects of electrons and Co-60 gamma rays on pBR322 plasmid DNA. Proc. 10th Int. Particle Accelerator Conf. (2019)

35. Herskind, C. et al. Biology of high single doses of IORT: RBE, 5 R's and other biological aspects. Radiat. Oncol. 12(24), 1-14 (2017).

36. Spadinger, I. \& Palcic, B. The relative biological effectiveness of ${ }^{60} \mathrm{Co} \gamma$-rays, $55 \mathrm{kVp} \mathrm{X}$-rays, $250 \mathrm{kVp} \mathrm{X}$-rays and $11 \mathrm{MeV}$ electrons at low doses. Int. J. Radiat. Biol. 61(3), 345-353 (1992).

37. Zackrisson, B. et al. Relative biological effectiveness of high-energy photons (up to $50 \mathrm{MV}$ ) and electrons (50 MeV). Radiat. Res. 128(2), 192-196 (1991).

38. Vysin, L. et al. Proton-induced direct and indirect damage of plasmid DNA. Radiat. Environ. Biophys. 54, 343-352 (2015).

39. Henthorn, N. T. et al. Clinically relevant nanodosimetric simulation of DNA damage complexity from photons and protons. RSC Adv. 9, 6845-6858 (2019).

40. Nikjoo, H. et al. Modelling of Auger-induced DNA damage by incorporated 125I. Acta Oncol. 35, 849-856 (1996).

41. Konstantinos, P. et al. Quantification of DNA double-strand breaks using Geant4-DNA. Med. Phys. 46(1), 405-413 (2018).

42. Ward, J. F. Biochemistry of DNA Lesions. Radiat. Res. 8(104), 103-111 (1985).

43. Hayes, F. The function and organisation of plasmids. in E. coli plasmid vectors. (Humana Press Inc., 2003) 1-18.

44. Leloup, C. et al. Evaluation of lesion clustering in irradiated plasmid DNA. Int. J. Radiat. Biol. 81, 41-54 (2005).

45. Lagzda, A., Angal-Kalinin, D., Jones, J. K., Jones, R. M. \& K. Kirkby. Relative insensitivity to inhomogeneities on very high energy electron dose distributions. Proc. 8th Int. Particle Accelerator Conf. (2017)

46. Lagzda, A. et al. Influence of heterogeneous media on very high energy electron (VHEE) dose penetration and a Monte Carlobased comparison with existing radiotherapy modalities. Nucl. Inst. Methods Phys. Res. B 482, 70-81 (2020).

47. GAFChromic EBT-XD film specifications, Available at www.gafchromic.com

48. Schindelin, J. et al. Fiji-an open platform for biological image analysis. Nat. Methods 9, 676-682 (2009).

49. Cowan, R., Collis, C. M. \& Grigg, G. W. Breakage of double-stranded DNA due to single-stranded nicking. J. Theor. Biol. 127, 227-245 (1987).

50. Agostinelli, S. et al. GEANT4-a simulation toolkit. Nucl. Inst. Methods Phys. Res. A 506, 250-303 (2003).

51. Incerti, S. et al. Geant4-DNA example applications for track structure simulations in liquid water: a report from the Geant4-DNA Project. Med. Phys. 45, 722-739 (2018).

52. Bernal, M. A. et al. Track structure modeling in liquid water: A review of the Geant4-DNA very low energy extension of the Geant4 Monte Carlo simulation toolkit. Phys. Med. 31, 861-874 (2015).

53. Incerti, S. et al. Comparison of Geant4 very low energy cross section models with experimental data in water. Med. Phys. 37, 4692-4708 (2010).

54. Incerti, S. et al. The Geant4-DNA project. Int. J. Model. Simul. Sci. Comput. 1, 157-178 (2010).

55. Sotiropoulos, M. et al. Modelling direct DNA damage for gold nanoparticle enhanced proton therapy. Nanoscale 9, 18413-18422 (2017).

56. Charlton, D. E., Nikjoo, H. \& Humm, J. L. Calculation of initial yields of single- and double-strand breaks in cell nuclei from electrons, protons and alpha particles. Int. J. Radiat. Biol. 56, 1-19 (1989).

57. McNamara, A. L. et al. Validation of the radiobiology toolkit TOPAS-nBio in simple DNA geometries. Phys. Med. 33, 207-215 (2016).

58. Bernal, M. A. \& Liendo, J. A. An investigation on the capabilities of the PENELOPE MC code in nanodosimetry. Med. Phys. 36, 620-625 (2009).

59. Prise, K. et al. Critical energies for SSB and DSB induction in plasmid DNA by low-energy photons: action spectra for strand-break induction in plasmid DNA irradiated in vacuum. Int. J. Radiat. Biol. 76, 881-890 (2000).

\section{Acknowledgements}

This research was co-funded by the Cockcroft Institute of Science and Technology/ASTeC STFC core grant and the EPSRC Grand Challenge Network+ in Proton Therapy (grant no. EP/N027167/1). In addition, R.M.J. is pleased to acknowledge support via an STFC IAA (Impact Acceleration Account) 2015 grant. N.T.H. would like to acknowledge financial support from the European Union's Horizon 2020 research and innovation programme under grant agreement no. 730983. M.J.M. would like to acknowledge some financial support from the NIHR Manchester Biomedical Research Centre (grant no. BRC-1215-20007). The authors acknowledge the GMEE for supporting the research of A.G.

\section{Author contributions}

Plasmid irradiation experiment was designed by K.L.S. with contributions to the conceptual development of the work by D.A-K., K.J.K., W.F., M.S., J.J., M.J.M. and R.M.J. Apparatus designed by K.L.S. and produced by R.J.S. Experiment carried out by K.L.S., N.T.H. and the CLEAR group (W.F., R.C., D.G., A.G.). Plasmid analysis carried out by K.L.S. and N.T.H., trained and supported by A.C. and E.S. EBT-XD film analysis carried out with A.A. Simulations performed by K.L.S. using code developed by N.T.H., K.L.S. and R.M.J. Supervision provided throughout by R.M.J. and M.J.M. K.L.S. wrote the main manuscript, with reviews by all authors.

\section{Competing interests}

The authors declare no competing interests.

\section{Additional information}

Supplementary Information The online version contains supplementary material available at https://doi. org/10.1038/s41598-021-82772-6.

Correspondence and requests for materials should be addressed to K.L.S.

Reprints and permissions information is available at www.nature.com/reprints.

Publisher's note Springer Nature remains neutral with regard to jurisdictional claims in published maps and institutional affiliations. 
(c) (i) Open Access This article is licensed under a Creative Commons Attribution 4.0 International cc) License, which permits use, sharing, adaptation, distribution and reproduction in any medium or format, as long as you give appropriate credit to the original author(s) and the source, provide a link to the Creative Commons licence, and indicate if changes were made. The images or other third party material in this article are included in the article's Creative Commons licence, unless indicated otherwise in a credit line to the material. If material is not included in the article's Creative Commons licence and your intended use is not permitted by statutory regulation or exceeds the permitted use, you will need to obtain permission directly from the copyright holder. To view a copy of this licence, visit http://creativecommons.org/licenses/by/4.0/.

(C) The Author(s) 2021 